\title{
71. Topology of Standard Path Spaces and Homotopy Theory. I
}

\author{
By Hirosi Toda \\ Department of Mathematics, Osaka City University \\ (Comm. by Z. Suetuna, M.J.A., July 13, 1953)
}

This is the first of a series of notes, whose aim is to clarify the homological structure of the path space $\Omega(X, A)=\left\{f: I^{1)} \rightarrow X\right.$ $\mid f(0)=*, f(1) \in A\}$ by means of "standard path space" and to investigate the homotopical structure of spaces. The paper of J-P. Serre ${ }^{2)}$ based on the singular homology theory of fibre spaces shows how the loop space $\Omega(X)=\Omega(X, *)$ is applied to the calculation of the Hurewicz homotopy groups $\pi_{p}(X)$ of $X$.

It was proved by J. B. Giever ${ }^{3)}$ that to every space $X$ there exists a CW-complex $P(X)$ and a map of $P(X)$ into $X$ inducing isomorphisms of the homotopy groups of $P(X)$ onto those of $X$. A problem to determine the homological structures of $P(\Omega(X))$ from those of $P(X)$ is closely related with Serre's theory. For the simply connected space $X$, this problem can be solved by selecting complexes $K(X)$ and $\omega(K(X))$, so-called a standard complex and a standard path complex respectively, when the complex $\omega(K(X))$ is combinatorially constructed from $K(X)$.

Here we give definitions of standard spaces and standard paths in them. The set of standard paths in a standard complex $K$, whose end points are in a subcomplex $L$ of $K$, forms a closed subset $\omega(K, L)$ of $\Omega(K, L)$. The standard path space $\omega(K, L)$ is a CW-complex and is constructed from $K$ and $L$ by a combinatiorial method.

The fundamental result in this note is roughly stated as follows; the injection: $\omega(K, L) \rightarrow \Omega(K, L)$ induces isomorphisms of homotopy and homology groups of $\omega(K, L)$ onto those of $\Omega(K, L)$.

Our theory is applied to determine the orders of homotopy groups $\pi_{p}\left(S^{n}\right)$ of $n$-sphere $S^{n}$ for $p \leqq n+8$.

$\S 1$. Standard Paths in a Suspended Space. Let $E(X)$ be a suspended space of a space $X$, which is obtained from $X \times I$ by shrinking a subset $* \times I \cup X \times I^{4}$ ) to a single point $*$, and let $d: X \times I \rightarrow E(X)$ be its shrinking map. Assume that a real function $\rho$ of $X$ is given such that $\rho$ is positive excepting $\rho(*)=0$. Then define a standard path $l\left(x_{1}, \ldots, x_{n} ; y, t\right): I \rightarrow E(X)$ by a formula

(A) $\quad l\left(x_{1}, \ldots, x_{n} ; y, t\right)(s)= \begin{cases}d\left(x_{i},\left(s-s_{i-1}\right) / \rho\left(x_{i}\right)\right) & s_{i-1} \leqq s \leqq s_{i} \\ d\left(y,\left(s-s_{n}\right) / \rho(y)\right) & s_{n} \leqq s \leqq 1,\end{cases}$

where $x_{i} \in X, y \in A<x, t \in I, s_{0}=0$ and $s_{i}=\sum_{k=1}^{i} \rho\left(x_{k}\right) /\left(\sum_{k=1}^{n} \rho\left(x_{k}\right)+t \cdot \rho(y)\right)$ 
for $i=1, \ldots, n$. The path $l\left(x_{1}, \ldots, x_{n} ; y, t\right)$ starts at the base point * and ends at a point $d(y, t)$ of $E(A)=d(A \times I)$. The set of paths $l\left(x_{1}, \ldots, x_{n} ; y, t\right)$ forms a closed subset $\omega(E(X), E(A))$ of the path space $\Omega(E(X), E(A))$, called a standard path space of the pair $(E(X)$, $E(A))$. Denote a loop $l\left(x_{1}, \ldots, x_{n_{2}} ; *, 0\right)$ by $l\left(x_{1}, \ldots, x_{n}\right)$ and denote the set $\omega(E(X), *)$ of standard loops by $\omega(E(X))$. Let $(X)^{n}$ be an $n$-fold product of $X$, identify $(X)^{n-1}$ to $(X)^{n-1} \times *<(X)^{n}$ and set $V_{n}$ $(X)^{n}=(X)^{\infty}$. Then the space $\omega(E(X), E(A))$ is obtained from $(X)^{\infty}$ $\times A \times I$ by the following identifications :

$\left(x_{1}, \ldots, x_{2} ; y, 0\right) \equiv\left(x_{1}, \ldots, x_{n-1} ; x_{n}, 1\right) \equiv\left(x_{1}, \ldots, x_{2 n} ; *, t\right)$ and $\left(x_{1}, \ldots, x_{n} ; y, t\right) \equiv\left(x_{1}, \ldots, x_{i-1}, x_{i+1}, \ldots, x_{n} ; y, t\right)$ if $x_{i}=*$.

Specially, the space $X$ is naturally imbedded into $\omega(E(X), E(A))$ by a correspondence: $x \rightarrow l(x)$.

A pair $(X, A)$ is said to have a homotopy extension property if $X \times(0) \cup A \times I$ is a deformation retract of $X \times I$. Then our primary result is ;

Theorem I. If $(X, A)$ and $(A, *)$ have the homotopy extension property and if $X$ is arcwise connected and $(X, A)$ is 1-connected, then the injection homomorphisms of homotopy groups $i_{*}: \pi_{p}(\omega(E(X))) \rightarrow$ $\pi_{p}(\Omega(E(X)))$ and $i_{*}: \quad \pi_{p}(\omega(E(X), E(A))) \rightarrow \pi_{p}(\Omega(E(X), E(A)))$ are all isomorphisms ${ }^{\mathrm{b}}$.

For a map $f:\left(Y, y_{*}\right) \rightarrow(\omega(E(X)), l(*))$ we define a suspension Ef: $E(Y) \rightarrow E(X)$ of $X$ by setting $E f(d(y, t))=f(y)(t)$. If $Y$ is a finite polyhedron, the homotopy classes of $f$ and $E f$ correspond one-to-one. Since the set of all classes of $E f$ coincides to the fundamental group of a function space $E(X)_{0}^{Y}=\left\{g: Y \rightarrow E(X) \mid g\left(y_{*}\right)=*\right\}$, the homotopy classes of $f$ form a group. This group is a generalization of the cohomotopy groups of E. Spanier ${ }^{\theta}$. We mention the fact that there are suspension isomorphisms

and

$$
\begin{aligned}
\pi_{p}(\omega(E(X))) & \approx \pi_{p+1}(E(X)), \\
\pi_{p}(\omega(E(X), E(A)) & \approx \pi_{p+1}(E(X), E(A)),
\end{aligned}
$$

where $X=d\left(X \times\left[\frac{1}{2}, 1\right]\right), \quad \hat{X}_{-}=d\left(X \times\left[0, \frac{1}{2}\right]\right)$ and $\pi_{p+1}\left(E(X) ; \hat{X}_{+}, \hat{X}_{-}\right)$is the homotopy groups of triad ${ }^{7}$.

$\$ 2$. Definition of Standard Paths. In this section we define a standard complex ${ }^{m} K=E\left(K_{0} ; f_{1}, E_{1} ; \ldots ; f_{m}, E_{m}\right)$ and standard paths in $K$ inductively. For $n=0,{ }^{\circ} K$ is the suspended space $E\left(K_{0}\right)$ of a $\mathrm{CW}$-complex $K_{0}$ and the standard path in it was already defined in $\S 1$ by the formula $(A)$ with respect to the function $\rho$ of $K_{0}$. Define a real function $\rho_{\mathrm{J}}$ of $\omega\left({ }^{0} K\right)$ by $\rho_{0}\left(l\left(x_{1}, \ldots, x_{n}\right)\right)=\rho\left(x_{1}\right)+\cdots+\rho\left(x_{n}\right)$. Suppose the standard complex ${ }^{m-1} K$, the space $\omega\left({ }^{m-1} K\right)$ of the standard loops in ${ }^{m-1} K$ and a real function $\rho_{m-1}$ of $\omega\left({ }^{m-1} K\right)$ are already 
defined such that ${ }^{m-1} K$ is a CW-complex and $\rho_{m-1}$ is positive excepting $\rho_{m-1}(l(*))=0$. Let $f_{m}$ be a map of $\left(S, s_{*}\right)$ into $\left(\omega\left({ }^{m-1} K\right), l(*)\right)$, where $S=\cup_{\alpha} S_{\alpha}^{n_{\alpha}}$ is the sum of $n_{\alpha}$-spheres $S_{\alpha}^{n_{\alpha} \alpha}\left(n_{\alpha} \geq 1\right)$ having a single point $s_{*}$ in common. Define a map $F_{m}: E(S) \rightarrow^{m-1} K$ by setting $F_{m}$ $(d(y, t))=f_{m}(y)(t)$, where $E(S)=\cup_{\alpha} S_{\alpha}^{n_{\alpha}+1}$ is the suspended space of $S$ and $d: S \times I \rightarrow E(S)$ is its shrinking map. Then the standard complex ${ }^{m} K={ }^{m-1} K \cup \bar{\varepsilon}_{m}=\left(\bar{\varepsilon}_{m} \cup_{\alpha} \varepsilon_{\alpha}^{n_{\alpha+2}}\right)$ is obtained from $K$ attaching the $\left(n_{\alpha}+2\right)$-cells $\varepsilon_{\alpha}^{n_{\alpha+2}}$ by $F_{m} \mid S_{\alpha}^{n_{\alpha}+1}$. Let $\omega\left({ }^{m-1} K\right) \cup \varepsilon_{m}\left(\varepsilon_{m}=\cup_{\alpha} \varepsilon_{\alpha}^{n_{\alpha+1}}\right)$ be a complex obtained from $\omega\left({ }^{m-1} K\right)$ attaching the $\left(n_{\alpha}+1\right)$-cells $\varepsilon_{\alpha}^{n_{\alpha}+1}$ by $f_{m} \mid s_{\alpha}^{n_{\alpha}}$, then there exists a map

$$
d_{m}:\left(\omega\left({ }^{m-1} K\right) \cup \varepsilon_{m}\right) \times I \rightarrow{ }^{m} K
$$

such that $d_{m}(l, t)=l(t)$ for $l \in \omega\left({ }^{m-1} K\right), d_{m}\left(\left(\omega\left(^{m-1} K\right) \cup \varepsilon_{m}\right) \times \dot{I}\right)=*$ and $d_{m}$ is homeomorphic elsewhere. The function $\rho_{m-1}$ of $\omega\left({ }^{m-1} K\right)$ is extendable to whole of $\varepsilon_{m}$ positively excepting $\rho_{m-1}(l(*))=0$. A standard path

$$
l\left(x_{1}, \ldots, x_{2} ; y, t\right): I \rightarrow^{m} K
$$

is defined for $\left.x_{i}, y \in \omega{ }^{m-1} K\right) \cup \varepsilon_{m}$ and $t \in I$ by the formula $(A)$, replacing the operations $d$ and $\rho$ by $d_{m}$ and $\rho_{m-1}$ respectively. The space of all standard loops $l\left(x_{1}, \ldots, x_{n}\right)=l\left(x_{1}, \ldots, x_{n} ; *, 0\right)$ will be denoted by $\omega\left({ }^{m} K\right)<\Omega\left({ }^{m} K\right)$. Finally we define a real function $\rho_{m}$ of $\omega\left({ }^{m} K\right)$ by $\rho_{m}\left(l\left(x_{1}, \ldots, x_{n}\right)\right)=\sum_{i=1}^{n} \rho_{m-1}\left(x_{i}\right)$.

In general, standard complex $K=E\left(K_{0} ; f_{1}, \varepsilon_{1} ; \ldots ; f_{m}, \varepsilon_{m} ; \ldots\right)$ is a CW-complex defined by $K=\cup_{m}^{m} K$. Since a correspondence $x \rightarrow l(x)$ $\left(x \in \omega\left({ }^{m-1} K\right)\right)$ is an imbedding of $\left.\omega{ }^{m-1} K\right)$ into $\left.\omega{ }^{m} K\right)$, we may define a space $\omega(K)$ of standard loops in $K$ by $\omega(K)=\cup_{m} \omega\left({ }^{m} K\right)$. Let $L$ be a subcomplex of $K$, then $L$ is represented by a form $E\left(L_{0} ; f_{1}^{\prime}, \varepsilon_{1}^{\prime}\right.$; $\left.\ldots ; f_{m}^{\prime}, \varepsilon_{m}^{\prime} ; \ldots\right)$ where $\varepsilon_{i}^{\prime}=\varepsilon_{i} \cap L$ and $f_{i}^{\prime}=f_{i} \mid \varepsilon_{i}^{\prime}$. The set of all paths $l\left(x_{1}, \ldots, x_{n} ; y, t\right)$, for $\left.x_{i} \in \omega{ }^{m-1} K\right) \cup \varepsilon_{m}, y \in \omega\left({ }^{m-1} L\right) \cup \varepsilon_{m}^{\prime}$ and $t \in I$, forms a colsed subset $\omega\left({ }^{m} K,{ }^{m} L\right)$ of $\Omega\left({ }^{m} K,{ }^{m} L\right)$ where ${ }^{m} L=L \cap^{m} K$. Let us define a space of standard paths $\omega(K, L)$ by $\cup_{m} \omega\left({ }^{m} K,{ }^{m} L\right)$. Then the space $\omega(K, L)$ is constructed from $K$ and $L$ combinatorially as follows, and this space becomes a CW-complex. Define a continuous map

$$
\vee: \quad \omega(K) \times \omega(K, L) \rightarrow \omega(K, L)
$$

by $\left.\vee(l(x)), l^{\prime}\left(x^{\prime}, t\right)\right)=l^{\vee} l^{\prime}\left(x ; x^{\prime}, t\right)$ for paths $x, x^{\prime}$ of ${ }^{m} K(m$; sufficiently large). Then $\vee\left(\omega\left({ }^{m} K\right) \times \omega\left({ }^{m} K\right)\right)=\omega\left({ }^{m} K\right), l^{\vee} l(*)=l(*)^{\vee} l=l$ and $l^{\vee}\left(l^{\prime} \vee l^{\prime \prime}\right)$ $=\left(l^{\vee} l^{\prime}\right)^{\vee} l^{\prime \prime}$, and this implies the simplicity of $\omega(K)$ and $(\Omega(K), \omega(K))$. Considering a correspondence $\left(x_{1}, \ldots, x_{n} ; y, t\right) \rightarrow l\left(x_{1}, \ldots, x_{2 n} ; y, t\right)$, we have that the standard path space $\omega\left({ }^{m} K,{ }^{m} L\right)$ is constructed from $\left.\left(\omega\left({ }^{m-1} K\right) \cup \varepsilon_{m}\right)^{\infty} \times\left(\omega{ }^{m-1} L\right) \cup \varepsilon_{m}^{\prime}\right) \times I$ by the following identifications ;

$$
\begin{aligned}
& \left(x_{1}, \ldots, x_{n} ; y, 0\right) \equiv\left(x_{1}, \ldots, x_{n-1} ; x_{n}^{\prime}, 1\right) \equiv\left(x_{1}, \ldots, x_{n} ; *, t\right), \\
& \left(x_{1}, \cdots, x_{n} ; y, t\right) \equiv\left(x_{1}, \cdots, x_{i-1}, x_{i+1}, \cdots, x_{n} ; y, t\right) \text { if } x_{i}=*,
\end{aligned}
$$


$\left(x_{1}, \ldots, x_{n} ; y, t\right) \equiv\left(x_{1}, \ldots, x_{i-1}, x_{i}^{\vee} x_{i+1}, x_{i+2}, \ldots, x_{n} ; y, t\right)$

if $x_{i}, x_{i+1} \in \omega\left({ }^{m-1} K\right)$ and

$$
\left(x_{1}, \ldots, x_{n} ; y, t\right) \equiv\left(x_{1}, \ldots, x_{n-1} ; x_{n} \vee l(y, t)\right)
$$

if $x_{n}, y \in \omega\left({ }^{m-1} K\right)$. This identifications offer us a cellular decomposition of $\omega\left({ }^{m} K,{ }^{m} L\right)$ and a fortiori that of $\omega(K, L)$. Cells of $\omega(K, L)$ are represented by finite sequences $\left(\sigma_{1}, \ldots, \sigma_{n} ; \tau\right)$ of cells $\sigma_{i} \in K$ and $\tau \in L$, and they have the dimensions; $\sum \operatorname{dim} \sigma_{i}+\operatorname{dim} \tau-n$.

Remark: Similar arguments may be treated for a standard space ${ }^{m} X=E\left(X_{0} ; f_{1}, \hat{X}_{1} ; \ldots ; f_{m}, \hat{X}_{m}\right)$, in which $\hat{X}_{i}$ are singular corns with bases $X_{i}$ and $f_{i}$ are maps of $X_{i}$ into standard loop spaces $\omega\left({ }^{i-1} X\right)$ of ${ }^{i-1} X$. The conclusion of the following theorem II holds under a suitable smoothness condition.

$\S 3$. Fundamental Theorem. The fundamental result of this notes is stated as follows :

Theorem II. If a pair $(K, L)$ of standard complexes is 2-connected and if $K$ is simply connected, then the injection homomorphisms of homotopy groups $i_{*}: \quad \pi_{p}(\omega(K)) \rightarrow \pi_{p}(\Omega(K))$ and $i_{*}: \pi_{p}(\omega(K, L)) \rightarrow \pi_{p}$ $(\Omega(K, L))$ are all isomorphisms.

Corollary. We have isomorphisms $\pi_{p}(\omega(K)) \approx \pi_{p+1}(K)$ and $\pi_{p}(\omega(K$, $L)) \approx \pi_{p+1}(K, L)$.

Lemma. For every simply connected space $X$, there exist a standard complex $K$ and a map $f$ of $K$ into $X$ inducing the isomorphisms of the homotopy groups of $K$ onto those of $X$.

Let $\pi_{p}\left(X ; X_{1}, \ldots, X_{n-1}\right)$ be the $n$-ad homotopy group of Blakers and Massey ${ }^{7)}$, and set $Y=X_{1} \cap \ldots \cap X_{n-1}$ and $Y_{i}=X_{1} \cap \ldots \cap X_{i-1} \cap$ $X_{i+1} \cap \ldots \cap X_{n-1}$. If $X=Y_{1} \cup \ldots \cup Y_{n-1}$, in the following exact sequence $\pi_{p+1}\left(X ; X_{1}, \ldots, X_{n-1}\right) \stackrel{\text { a }}{\rightarrow} \pi_{p}\left(X_{1} ; X_{1} \cap X_{2}, \ldots, X_{1} \cap X_{n-1}\right) \stackrel{\text { i* }}{\rightarrow} \pi_{p}$ $\left(X ; X_{2}, \ldots, X_{n-1}\right) \rightarrow \pi_{p}\left(X ; X, \ldots, \dot{X}_{n-1}\right) \stackrel{\partial}{\rightarrow} \cdots$, the injection homomorphism $i_{*}$ is an excision of $(n-1)$-ad homotopy group, because $X_{1} \cap X_{i}=X_{i}-\left(Y_{1}-Y\right)(i=2, \ldots, n-1)$. Then the main theorem of Blakers and Massey ${ }^{7)}$ in triad homotopy group is generalized to

Proposition 1. Let $\left(X ; X_{1}, \ldots, X_{n-1}\right)$ be an $n$-ad such that $X=$ $Y_{1} \cup \ldots \cup Y_{n-1} \quad$ If $X$ is simply connected and $\left(Y_{i}, Y\right)$ are $r_{i}$-connected $\left(r_{i} \geqq 2\right)$ and if in every subpair of $\left(X ; X_{1}, \ldots ; X_{n-1}\right)$ the excision axiom of singular homology theory holds, then we have

$$
\pi_{p}\left(X ; X_{1}, \ldots, X_{n-1}\right)=0 \quad \text { for } n \leqq p \leqq \sum_{i=1}^{n-1} r_{i} .
$$

Let $K^{*}=K \cup \varepsilon^{n}$ be a complex obtained from a complex $K$ by attaching the singular $n$-cells. If $\left(K, \dot{\varepsilon}^{n}\right)$ is $m$-connected and $\dot{\varepsilon}^{n}$ is $r$-connected then homomorphisms

$$
P: \pi_{n}\left(\varepsilon^{n}, \dot{\varepsilon}^{n}\right) \times \pi_{p-n+1}\left(X, \dot{\varepsilon}^{n}\right) \rightarrow \pi_{p}\left(X^{*} ; \varepsilon^{n}, X\right)
$$

induced by the generalized Whitehead product ${ }^{8)}$ are isomorphisms for $p<m+n+r$ and homomorphisms onto for $p \leqq m+n+r$. 
§4. Homotopy Groups of Sphers. Since the $(n+1)$-sphere $S^{n+1}$ is a suspended space of the $n$-sphere $S^{n}$, the standard loop space $\omega\left(S^{n+1}\right)$ may be defined, and it is constituted by $k n$-cells $e^{k n} \quad(k=0$, $1,2, \ldots)$ such that $e^{\prime} \cup e^{n}$ is an $n$-sphere $S^{n}$ and $e^{2 n}$ is attached to $S^{n i}$ by a map $\left[i_{n}, i_{n}\right]: S^{2 n-1} \rightarrow S^{n}$ which represents Whitehead product of the identical map of $S^{n}$. The attachment of $e^{c_{n}}$ is represented by means of the generalized Whitehead product ${ }^{8)}\left(2+(-1)^{n}\right)\left[i_{n}, i_{2_{n}}\right]_{r}$ and a nullhomotopy of $\partial\left(\left(2+(-1)^{n}\right)\left[i_{n}, i_{\aleph_{n}}\right]_{r}\right)=\left(2+(-1)^{n}\right)\left[i_{n},\left[i_{n}, i_{n}\right]\right]$, where $i_{\Omega_{n}}$ represents a generator of $\pi_{\Sigma_{n n}}\left(S^{n} \cup e^{n n}, S^{n}\right)$.

Proposition 2. There are homomorphisms $\chi$ of $\pi_{p}\left(S^{2 n-1}\right)+\pi_{p}$ $\left(S^{3 n-2} \smile_{3} e^{n n-1}\right)$ for even $n\left(\pi_{p}\left(S^{2 i n-1}\right)\right.$ for odd $\left.n\right)$ into $\pi_{p+2}\left(S^{n+1} ; E_{+}^{n+1}, E_{-}^{n+1}\right)$ such that $\chi$ are isomorphisms for $p<4 n-3$ and homomorphisms onto for $p \leqq 4 n-3$, where $S_{3}^{3 n-2} e^{n n-1}$ is a cell-complex obtained from $S^{: n-2}$ attaching a cell $e^{n-1}$ by a mapping of degree 3 .

By normalizing the complex $\omega\left(S^{n+1}\right)$ to a standard form, we denote a standard loop complex $\omega\left(\omega\left(S^{n+1}\right), S^{n}\right)$ by $Q_{n+1}$. Then the homology groups of $Q_{n+1}$ are applied to the calculation of the homotopy groups $\pi_{p}\left(Q_{n+1}\right) \approx \pi_{p+2}\left(S^{n+1} ; E_{+}^{n+1}, E_{-}^{n+1}\right)$. In case $n=3$, we have the following results;

\begin{tabular}{c|c|c|c|c|c|c|c|c}
\hline$P$ & 3 & 4 & 5 & 6 & 7 & 8 & 9 & 10 \\
\hline$H_{p}\left(Q_{3}\right)$ & $Z$ & $Z_{3}$ & 0 & $Z_{2}$ & $Z_{3}$ & $Z_{15}$ & $Z_{2}$ & 0 \\
\hline$\pi_{p}\left(Q_{3}\right)$ & $Z$ & $Z_{6}$ & $Z_{2}$ & $Z_{24}$ & $Z_{6}$ & $Z_{30}$ & $Z_{6}$ & \\
\hline
\end{tabular}

It follows from an exact sequence

that

$$
\cdots \rightarrow \pi_{p}\left(Q_{n+1}\right) \rightarrow \pi_{p}\left(S^{n}\right) \stackrel{E}{\rightarrow} \pi_{p+1}\left(S^{n+1}\right) \rightarrow \pi_{p-1}\left(Q_{n+1}\right) \rightarrow \cdots
$$

Proposition 3. We have $\pi_{9}\left(S^{3}\right)=Z_{3}, \pi_{10}\left(S^{3}\right)=Z_{15}$ and $\pi_{11}\left(S^{3}\right)=Z_{\text {a }}$.

Corollary9) i) $\pi_{10}\left(S^{4}\right)=Z_{24}+Z_{3}$, and $\pi_{n+6}\left(S^{n n}\right)=Z$ for $n \geqq 5$.

ii) $\pi_{11}\left(S^{4}\right)=Z_{15}, \pi_{12}\left(S^{5}\right)=\pi_{13}\left(S^{6}\right) / Z_{2}=Z_{30}$, $\pi_{14}\left(S^{7}\right) / G_{4}{ }^{10)}=Z_{30}, \pi_{15}\left(S^{8}\right)=Z+\pi_{14}\left(S^{7}\right)$ and $\pi_{n+7}\left(S^{2 n}\right) / G_{4}=Z_{60}$ for $n \geqq 9$.

iii) $\pi_{12}\left(S^{4}\right)=\pi_{13}\left(S^{5}\right)=Z_{2}, \pi_{14}\left(S^{6}\right) / Z_{2}=Z_{2}$,

$\pi_{15}(S) / Z_{2}=Z_{2}, \pi_{10}\left(S^{8}\right)=Z_{2}+\pi_{15}\left(S^{7}\right), \pi_{17}\left(S^{9}\right) / Z_{2}=Z_{2}$ and $\pi_{n+8}\left(S^{n}\right)=Z_{2}$

for $n \geq 10$.

\section{References}

1) $I=[0,1]$ indicates unit interval.

2) "Homologie singulière des espace fibrés", Ann. Math., 54 (1951). (1950).

3) "On the equivalence of two singular homology theories", Ann. Math., 51

4) $\dot{I}=(0) \smile(1)$. 
5) The theorem holds for the injection homomorphisms of the singular homology groups.

6) “Borsuk's cohomotopy groups", Ann. Math., 50 (1949).

7) A. L. Blakers and W.S. Massey: "The homotopy groups of a triad I", Ann. Math., 53 (1952).

8) H. Toda: "Generalized Whitehead products and homotopy groups of spheres", Jour. Inst. Poly. Osaka City Univ., 3 (1952).

9) For $p \leqq n+5$, the groups $\pi_{p}\left(S^{\prime \prime}\right)$ are obtained by J-P. Serre ("Sur la suspension de Freudenthal ", C. R., 234 (1952)) and the author.

10) $G_{4} / Z_{2}=Z_{2}$ 